OPEN ACCESS

Edited by:

David William Waite,

University of Auckland, New Zealand

Reviewed by:

Yong Su,

Nanjing Agricultural University, China

Hua Yang,

ZheJiang Academy of Agricultural

Sciences, China

Devin B. Holman,

Agriculture and Agri-Food Canada,

Canada

*Correspondence:

Junjun Wang

jkywjj@hotmail.com

Specialty section:

This article was submitted to

Microbial Symbioses,

a section of the journal

Frontiers in Microbiology

Received: 09 April 2018

Accepted: 17 July 2018

Published: 07 September 2018

Citation:

Li N, Huang S, Jiang L, Wang W, Li T,

Zuo B, Li Z and Wang J (2018)

Differences in the Gut Microbiota

Establishment and Metabolome

Characteristics Between Lowand Normal-Birth-Weight Piglets

During Early-Life.

Front. Microbiol. 9:1798.

doi: 10.3389/fmicb.2018.01798

\section{Differences in the Gut Microbiota Establishment and Metabolome Characteristics Between Low- and Normal-Birth-Weight Piglets During Early-Life}

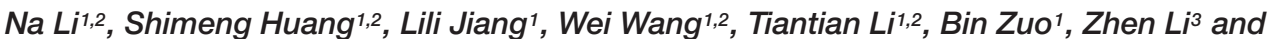 \\ Junjun Wang ${ }^{1,2 *}$
}

\footnotetext{
'State Key Laboratory of Animal Nutrition, College of Animal Science and Technology, China Agricultural University, Beijing, China, ${ }^{2}$ Beijing Advanced Innovation Center for Food Nutrition and Human Health, China Agricultural University, Beijing, China, ${ }^{3}$ State Key Laboratory of Plant Physiology and Biochemistry, College of Biological Sciences, China Agricultural University, Beijing, China
}

Low-birth-weight (LBW) piglets are at a high-risk for postnatal growth failure, mortality, and metabolic disorders later in life. Early-life microbial exposure is a potentially effective intervention strategy for modulating the health and metabolism of the host. Yet, it has not been well elucidated whether the gut microbiota development in LBW piglets is different from their normal littermates and its possible association with metabolite profiles. In the current study, $16 \mathrm{~S}$ rRNA gene sequencing and metabolomics was used to investigate differences in the fecal microbiota and metabolites between LBW and normal piglets during early-life, including day 3 (D3), 7 (D7), 14 (D14), 21 (D21, before weaning), and 35 (D35, after birth). Compared to their normal littermates, LBW piglets harbored low proportions of Faecalibacterium on D3, Flavonifractor on D7, Lactobacillus, Streptococcus, and Prevotella on D21, as well as Howardella on D21 and D35. However, the abundance of Campylobacter on D7 and D21, Prevotella on D14 and D35, Oscillibacter and Moryella on D14 and D21, and Bacteroides on D21 was significantly higher in LBW piglets when compared with normal piglets. The results of the metabolomics analysis suggested that LBW significantly affected fecal metabolites involved in fatty acid metabolism (e.g., linoleic acid, $\alpha$-linolenic acid, and arachidonic acid), amino acid metabolism (e.g., valine, phenylalanine, and glutamic acid), as well as bile acid biosynthesis (e.g., glycocholic acid, 25-hydroxycholesterol, and chenodeoxycholic acid). Spearman correlation analysis revealed a significant negative association between Campylobacter and N1-acetylspermine on D7, Moryella and linoleic acid on D14, Prevotella and chenodeoxycholic acid on D21, and Howardella and phenylalanine on D35, respectively. Collectively, LBW piglets have a different gut bacterial community structure when compared with normal-birth-weight (NBW) piglets during early-life, especially from 7 to 21 days of age. Also, a distinctive metabolic 
status in LBW piglets might be partly associated with the altered intestinal microbiota. These findings may further elucidate the factors potentially associated with the impaired growth and development of LBW piglets and facilitate the development of nutritional interventions.

Keywords: low-birth-weight, fecal metabolites, microbiota, metabolite, piglet

\section{INTRODUCTION}

Genetic selection for high-prolific sows has substantially increased litter size over the last few decades (Yuan et al., 2015). However, larger litters are closely correlated with an increasing prevalence of LBW piglets (Quesnel et al., 2008) due to IUGR (Wu et al., 2006). Piglets with a birth weight of less than $1.1 \mathrm{~kg}$ are defined as LBW, which accounts for $15-25 \%$ of neonatal piglets (Li et al., 2017; Wang et al., 2017). LBW piglets are more prone to neonatal deaths, postnatal growth restriction, as well as poor carcass quality (Wu et al., 2006; Berard et al., 2008). In addition, long-term dysfunctions in vital organs are observed in LBW piglets, especially the impaired development of the GIT (Wang et al., 2008, 2014; Li et al., 2017).

The GIT in mammals harbors a large microbial community (Rooks and Garrett, 2016). The early-life development of the gut microbiota is believed to be paramount for the early-stage maturation of gut barrier function, the innate immune system, and the health of the host (Matamoros et al., 2013; Kabat et al., 2014). The gut microbiota in neonates is extremely turbulent, and it is shaped by many environmental factors, including host genetics (Goodrich et al., 2014), delivery mode (Wang et al., 2013), dietary change (Li et al., 2012; Bian et al., 2016), and feeding environment (Inman et al., 2010; Schokker et al., 2014). A dysbiosis in the gut microbial community of neonates not only results in a higher risk of diseases but also causes short- and longlasting adverse effects on health (Foxx-Orenstein and Chey, 2012; Saavedra and Dattilo, 2012).

Many studies have reported the early-life development of the gut microbiota in newborn piglets born with NBW (Frese et al., 2015; Bian et al., 2016; Hu et al., 2016; Chen et al., 2017; Mu et al., 2017), while few studies have focused on the gut bacterial succession in LBW piglets during early-life. Previous studies have shown that counts of adherent bacteria via a traditional colony-counting method were greater in the intestinal mucosa of 2- to 5-day-old piglets with IUGR, when compared with the normal ones (D'Inca et al., 2010, 2011). However, these studies did not characterize the taxonomic composition of the bacterial community or assess its dynamic changes during the early-life of piglets. Therefore, differences in the gut microbiota of LBW

Abbreviations: ADG, average daily gain; FDR, false discovery rate; FXR, farnesoid X receptor; GIT, gastrointestinal tract; GLM, general linear model; GPBAR1, G-protein-coupled bile acid receptor 1; HESI, heated electrospray ionization; IUGR, intrauterine growth restriction; LBW, low-birth-weight; LDA, linear discriminant analysis; LEfSe, linear discriminant analysis effect size; NBW, normal-birth-weight; OPLS-DA, orthogonal partial least squares discriminant analysis; OTUs, operational taxonomic units; PCoA, principal coordinates analysis; PERMANOVA, permutational multivariate analysis of variance; SCFAs, shortchain fatty acids; SEM, standard error of the mean; UPLC-MS, ultrahigh performance liquid chromatography-mass spectroscopy. and NBW piglets in the early stages after birth need to be further studied.

One of the mechanisms through which the gut microbiota indirectly impacts host physiology is the production of microbial metabolites and modulation of host immunity (Rooks and Garrett, 2016; Turroni et al., 2018). Metabolic disorders caused by the gut microbiota are often associated with disease such as insulin resistance, diabetes, and inflammation (Zmora et al., 2017; Turroni et al., 2018). For example, metabolomic alterations were observed in the small intestine of 21-day-old piglets suffering from IUGR (He et al., 2011). To our knowledge, information about the changes in intestinal microbiota-related metabolites in LBW piglets is not available.

We hypothesized that the gut microbiota development and metabolism in LBW piglets were different from their normal littermates during early-life. Therefore, the present study was designed to investigate differences in the gut microbiota composition and fecal metabolome between LBW and NBW piglets during early-life, including the preweaning and postweaning period. Possible associations between microbes and metabolites were also revealed. The results of this study will help to further understand the negative effects on growth performance in LBW piglets and facilitate the exploration of new therapeutic biomarkers for newborns with LBW.

\section{MATERIALS AND METHODS}

\section{Ethics Statement}

All experimental protocols were carried out with the approval of the China Agricultural University Animal Care and Use Committee (CAU20170114-1, Beijing, China).

\section{Animal Management and Fecal Sampling}

In this study, a total of 30 multiparous sows (Yorkshire; $2 \sim 4$ parities) were selected and raised individually in a commercial pig breeding farm in Sichuan province, China. During the entire experimental period, sows were fed the same commercial feed and water was provided ad libitum from nipple drinkers. Thirty litters of piglets (Landrace $\times$ Yorkshire) were spontaneously delivered from sows after 113 114 days of gestation. At birth, $1 \mathrm{LBW}$ piglet $(0.75 \sim 0.95 \mathrm{~kg})$ and 1 NBW piglet $(1.35 \sim 1.55 \mathrm{~kg})$ were obtained from each of the thirty litters (each litter with $12 \sim 14$ piglets). No cross-fostering was used in this study. From day 3 to 5 after birth, piglets started to receive commercial creep feed and drinking water ad libitum. All the piglets were weaned at 21 days of age and transferred into the nursery pens with free access to 
commercial weaning diet and water. None of the piglets were administered with antibiotics or other drugs throughout this experiment.

Body weights of all newborn piglets were recorded immediately after delivery. On day 3 (D3), 7 (D7), 14 (D14), 21 (D21, before weaning), and 35 (D35, after birth), piglets (6 LBW and $6 \mathrm{NBW}$ piglets) from each of the six litters were weighed individually after $2 \sim 4 \mathrm{~h}$ of fasting and then sacrificed after anesthesia for sample collection. To avoid contamination, fresh feces were collected from the terminal rectum of each piglet. A total of 60 fecal samples from the rectum were collected on ice, immediately frozen in liquid nitrogen, and then stored at $-80^{\circ} \mathrm{C}$ until microbiome and metabolome analysis.

\section{DNA Extraction, 16S rRNA Gene Amplification and Sequencing}

Total metagenomic DNA was extracted from $200 \mathrm{mg}$ of each fecal specimen by using the QIAamp ${ }^{\circledR}$ Fast DNA Stool Mini Kit (Qiagen Ltd., Germany) in accordance with manufacturer's instructions. The V3-V4 region of the 16S rRNA gene was amplified with universal primers 341F (ACTCCTACGGGAGGCAGCAG) and 806R (GGACTACHVGGGTWTCTAAT), as described by Hong et al. (2016). The amplified products were detected using agarose gel electrophoresis (2\% agarose), recovered by AxyPrep DNA Gel Recovery Kit (Axygen Biosciences, Union City, CA, United States), and then quantified by Qubit 2.0 Fluorometer (Thermo Fisher Scientific, Waltham, MA, United States) to pool into equimolar amounts. Amplicon libraries were sequenced on the Illumina HiSeq 2500 platform (Illumina, San Diego, CA, United States) for paired-end reads of $250 \mathrm{bp}$. All the raw data involved in the present study were deposited in NCBI Sequence Read Archive (SRA) under accession number SRP137635.

\section{Analysis of Sequencing Data}

The raw paired-end reads were assembled into longer sequences and quality filtered by PANDAseq (version 2.9) to remove the low-quality reads with a length of $<220$ nucleotides (nt) or $>500 \mathrm{nt}$, an average quality score of $<20$, and sequences containing $>3$ nitrogenous bases (Masella et al., 2012). The high-quality sequences were clustered into OTUs with a 97\% similarity using UPARSE (version 7.0) (Edgar, 2013) in QIIME (version 1.8) (Caporaso et al., 2010), and the chimeric sequences were removed using UCHIME (Edgar et al., 2011). Taxonomy was assigned to OTUs using the RDP classifier ${ }^{1}$ (Bacci et al., 2015) against the SILVA 16S rRNA gene database (Release1282 ${ }^{2}$ ) (Pruesse et al., 2007), with a confidence threshold of $70 \%$.

The Shannon diversity index and the number of OTUs per sample were calculated by the MOTHUR program (version v.1.30.1 ${ }^{3}$ ) (Schloss et al., 2009). Bar plots and heat maps were generated with the "vegan" package in R (version 3.3.1). For beta

\footnotetext{
${ }^{1}$ http://rdp.cme.msu.edu/

${ }^{2}$ http://www.arb-silva.de

${ }^{3}$ http://www.mothur.org
}

diversity analysis, PCoA was performed based on Bray-Curtis distances using QIIME (version 1.8).

\section{Fecal Metabolite Extraction and UPLC-MS Analysis}

A total of 60 fecal samples were analyzed in the UPLC-MS platform according to the protocol described in a previous study (Cao et al., 2016). In brief, each fecal sample ( 100 mg) was mixed with $400 \mu \mathrm{L} \mathrm{MeOH}: \mathrm{ACN}\left(1: 1, \mathrm{v} / \mathrm{v}, 4^{\circ} \mathrm{C}\right)$, followed by centrifugation at $15,000 \times g$ for $10 \mathrm{~min}$. The supernatant was transferred to another $1.5 \mathrm{~mL}$ centrifuge tube and dried in a vacuum concentrator (Concentrator plus, Eppendorf). Next, the dried extracts were separately redissolved with $200 \mu \mathrm{L}$ $\mathrm{MeOH}: \mathrm{H}_{2} \mathrm{O}\left(4: 1, \mathrm{v} / \mathrm{v}, 4^{\circ} \mathrm{C}\right)$, and then centrifuged at $15,000 \times g$ for $10 \mathrm{~min}$. The final supernatant was filtered through a $0.22 \mu \mathrm{m}$ sterile membrane and proceeded using a UPLC-HRMS system (UPLC, ACQUITYUPLC H-Class Bio, Waters; MS, Q-Exactive, Thermo Scientific), equipped with a HESI source under the standard procedures.

\section{Metabolomics Data Processing}

Raw data processing and further data analysis were conducted according to a previous publication (Cao et al., 2016). In brief, SIEVE 2.1 software (Thermo Fisher Scientific, NJ, United States) was applied for peak alignment, background exclusion, and component extraction of raw data. Component extraction was achieved between retention times 0.5 and $16 \mathrm{~min}$, with intensity threshold at 500,000, minimum scan at 9, and signal-to-noise ratio of 10. OPLS-DA was generated using SIMCA-P 13 software (Umetrics, Umea, Sweden) after data were scaled to Pareto variance. Compounds with a criterion of CV $<20 \%$, fold change $>1.5$, and $P<0.05$ were filtered as differential metabolites between two groups, using EXCEL for further identification. The identification of these candidate metabolites was carried out using the Human Metabolome Database ${ }^{4}$ and METLIN ${ }^{5}$ based on the exact masses of molecular ions. The MS/MS spectra database was used to match fragment ion spectra of the candidate compounds. Similarly, MS/MS spectra were also compared with theoretical fragmentation patterns with mass tolerance at 5 ppm using Xcalibur ${ }^{\mathrm{TM}}$ (Thermo Fisher Scientific, NJ, United States). The impacts of birth weight on metabolic pathways and metabolite set enrichment analysis (MSEA) were analyzed using MetaboAnalyst $4.0^{6}$ (Chong et al., 2018).

\section{Statistical Analysis}

The difference in growth performance between LBW and NBW piglets at different time-points was tested using the GLM (SPSS 20.0). Both age and birth weight were considered as fixed factors, and means were separated and adjusted using Duncan's multiple test. $P$-values below 0.05 were considered statistically significant. The difference in the alpha diversity between LBW and NBW piglets at each time-point was tested using Mann-Whitney $U$-test (SPSS 20.0), and $P$-values were adjusted with FDR (below 5\%)

\footnotetext{
${ }^{4}$ http://www.hmdb.ca

${ }^{5}$ http://metlin.scripps.edu

${ }^{6}$ http://www.metaboanalyst.ca
} 
as described by Benjamini and Hochberg (1995). The corrected $P$-values below 0.05 were regarded as statistically significant. To compare the difference in the gut microbiota structure between LBW and NBW piglets at different time-points, PERMANOVA (1, 000 Monte Carlo permutations) was performed based on Bray-Curtis distances with the Adonis function available in the package "vegan" in R (version 3.3.1) (Anderson and Walsh, 2013). Linear discriminant analysis (LDA) effect size (LEfSe) analysis was used to identify the differential genera between LBW and NBW groups. Only genera with an average relative abundance greater than $0.01 \%$ were considered. Correlations between different metabolites and bacterial communities were assessed by Spearman's correlation analysis using the "pheatmap" package in $\mathrm{R}$ (version 3.3.1). Data were expressed as mean values.

\section{RESULTS}

\section{Effects of Birth Weight on Growth \\ Performance in Piglets}

Low-birth-weight piglets continuously showed a significantly lower body weight $(P<0.001)$ than NBW piglets during the whole experimental period (Supplementary Table S1). At D35, the body weight of LBW piglets was $17 \%$ lower than that of the normal ones. Furthermore, from D3 to D21, LBW piglets had a lower ADG when compared with NBW piglets $(P<0.001)$.

\section{Summary of 165 rRNA Gene Profiles and Alpha Diversities Across All the Samples}

A total of 3, 136, 875 high-quality $16 \mathrm{~S}$ rRNA gene sequences were generated from sixty fecal samples. We randomly subsampled all the samples to 30, 996 sequences to avoid bias caused by different sequencing depth. Based on $97 \%$ sequence similarity,
1, 076 OTUs were identified and then assigned to 17 phyla, 32 classes, 54 orders, 89 families, and 264 genera.

The difference in gut bacterial diversity and richness between LBW and NBW piglets is shown in Figure 1. Birth weight had no significant influence on fecal bacterial richness and diversity (number of OTUs and Shannon diversity index) at any tested time-point (Figures 1A,B).

\section{Age-Induced Changes in the Gut Microbiota of Piglets From Preweaning to Postweaning Period}

The overall bacterial composition of the piglet gut varied significantly by age, irrespective of birth weight (Figure 2 and Supplementary Figure S1). A PCoA plot of the BrayCurtis distances confirmed that samples clustered primarily in an age-dependent manner along the PC1 axis (Figure 3). A PERMANOVA of these distances also showed that the gut microbiota structure was strongly affected by age $\left(R^{2}=0.382\right.$, $P=0.001$ ). At the phylum level (Supplementary Figure S1), Bacteroidetes was predominant at all time-points, with the mean relative abundance ranging from $42.0-51.9 \%$. The relative abundance of Firmicutes was lower on D3 (16.8\%) when compared to D7 (36.1\%). Fusobacteria and Proteobacteria were the dominant phyla on D3 but the levels decreased on D7. Furthermore, Fusobacteria was nearly undetectable on D21 and D35. At the genus level (Figure 2), Bacteroides, Fusobacterium, and Escherichia-Shigella were the main bacterial genera in the fecal samples from D3 to D7. In contrast, Prevotella, Phascolarctobacterium, Prevotellaceae NK3B3E group and Alloprevotella were dominant in the gut from D14 to D35. Lactobacillus had a lower relative abundance on D3 $(1.6 \%)$ but was predominant at other ages, with the highest value on D7 (18.7\%). The relative abundances of the top 50 most abundant genera are shown in Supplementary Figure S2.

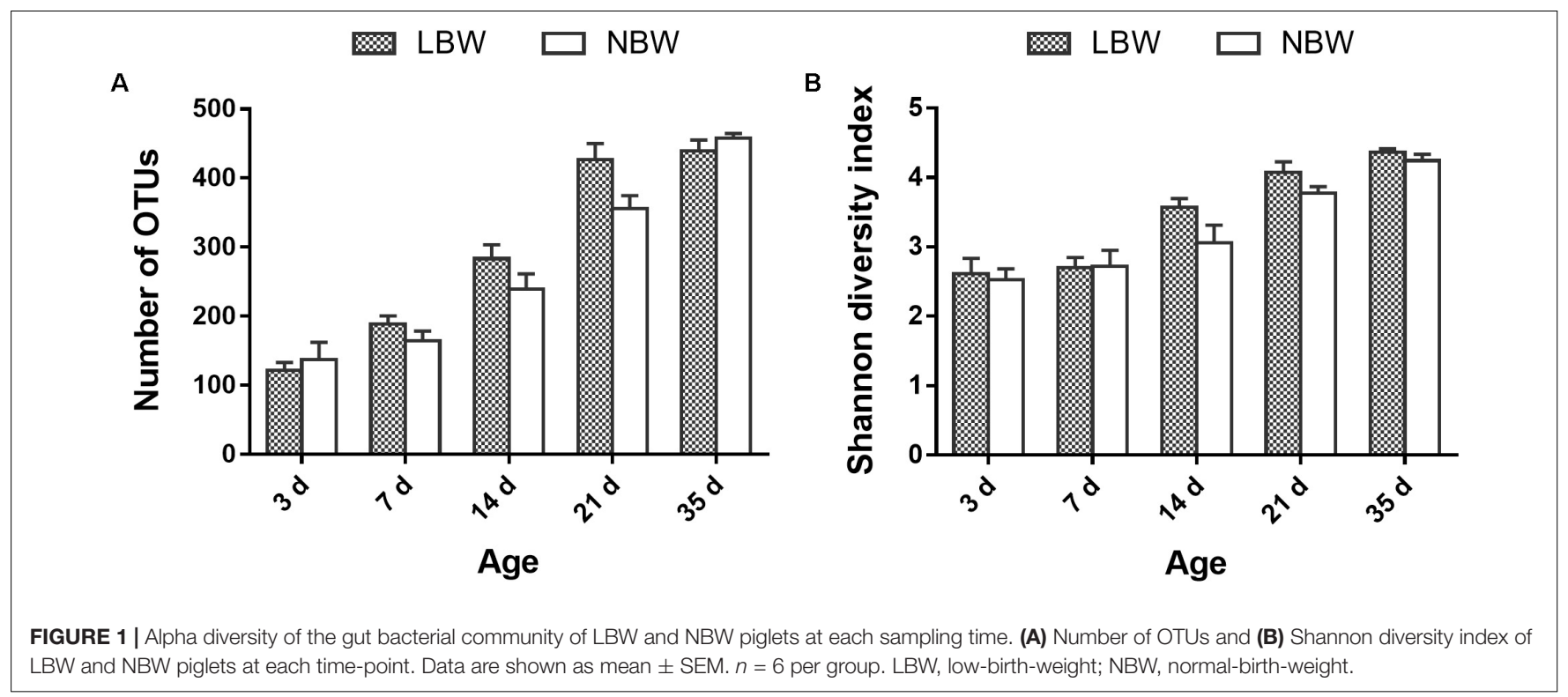



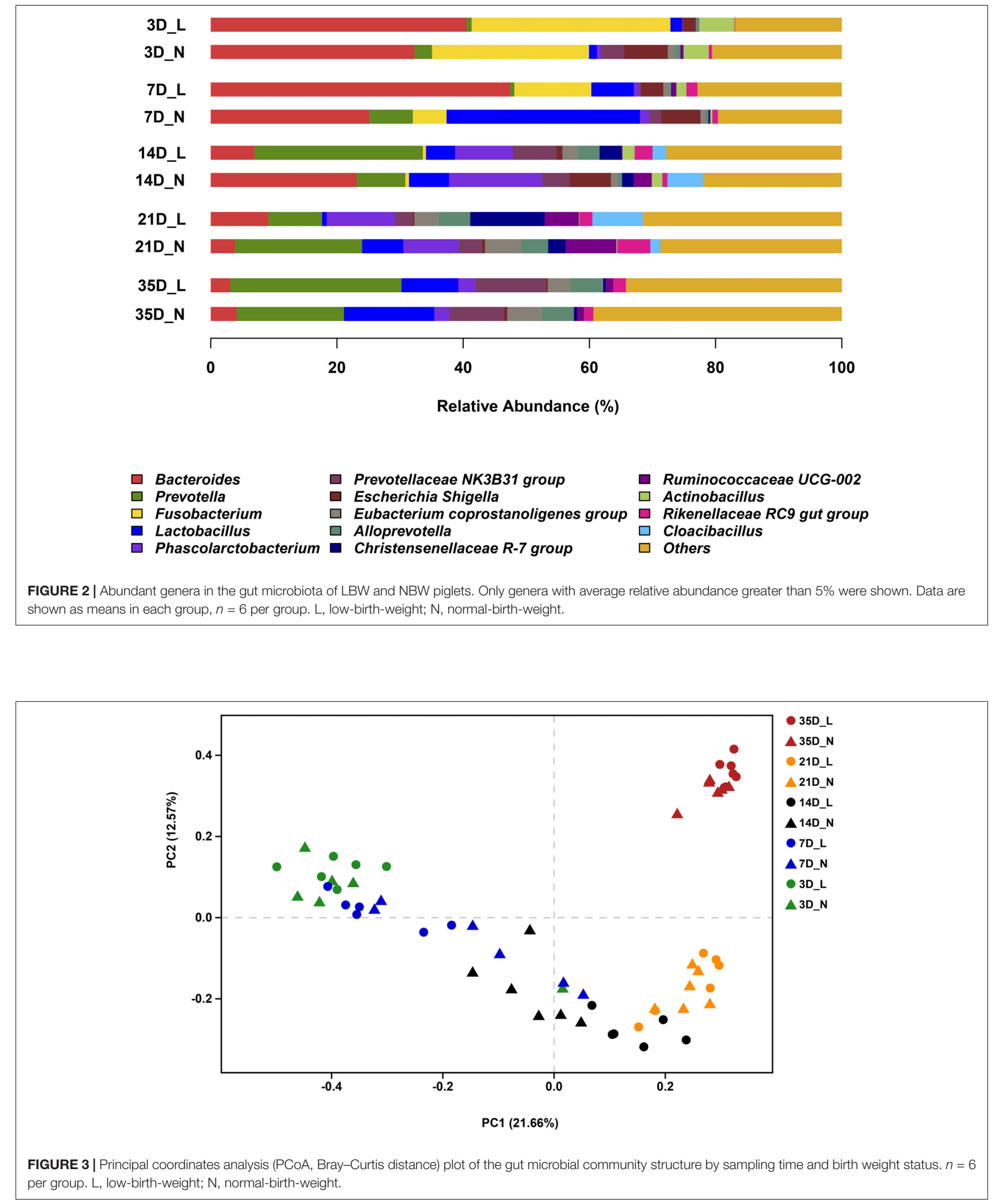


\section{Differences in the Gut Microbiota Between LBW and NBW Piglets During Early-Life}

We visualized the Bray-Curtis distances using PCoA and statistically with PERMANOVA to evaluate the effect of birth weight status on the bacterial community structure. The PCoA plot revealed distinct separation of the bacterial community structure by birth weight on D7, D14, and D21 (Supplementary Figures S3B,C,D), which was confirmed by PERMANOVA (D7, $R^{2}=0.182, P=0.009 ; \mathrm{D} 14, R^{2}=0.169$, $P=0.012$; D21, $\left.R^{2}=0.157, P=0.017\right)$. However, the microbial community structure was relatively similar between LBW and NBW piglets on D3 (Supplementary Figure S3A; $R^{2}=0.145$, $P=0.084$ ) or D35 (Supplementary Figure S3E; $R^{2}=0.116$, $P=0.077)$.

Significant differences in the relative abundance of phyla and genera in the fecal microbiota between LBW and NBW piglets at a certain age were further identified using the Mann-Whitney $U$-test and LEfSe analysis (Figure 4, Supplementary Figure S4, and Supplementary Table S2). As the most dominant phylum, the relative abundance of the Bacteroidetes between LBW and NBW piglets was not significantly different at any time-point $(P>0.05$, Supplementary Figure S4B). Compared with the NBW group, the relative abundance of the phylum Firmicutes was significantly lower $(P<0.05$, Supplementary Figure S4A) in LBW piglets on D3 (mean, $11.9 \%$ vs. $21.7 \%$ ) and D7 (mean, $25.9 \%$ vs. $46.2 \%$ ). In contrast, the relative abundance of the phylum Fusobacteria (and the genus Fusobacterium) in LBW piglets was relatively high on D3 and D7, but the difference was not statistically significant $(P>0.05$, Supplementary Figures S4C,G).

Results of LEfSe at the genus level revealed that one taxon on D3 was significantly impacted by birth weight, followed by three taxa on D7, eleven taxa on D14, seventeen taxa on D21, and six taxa on D35 (Figure 4 and Supplementary Table S2). For example, on D3, LBW piglets showed a dramatically lower relative abundance of the genus Faecalibacterium $(0.001 \%$ vs. $0.018 \%, P<0.05)$ than NBW piglets. On D7, the relative abundances of the genus Campylobacter $(0.074 \%$ vs. $0.001 \%$, $P<0.05)$ and Ruminococcaceae UCG-005 (0.059\% vs. $0.003 \%$, $P<0.05)$ were higher in LBW piglets, while a lower relative abundance of Flavonifractor $(0.063 \%$ vs. $0.314 \%, P<0.05)$ was observed in NBW piglets. A lower proportion of the genus Escherichia-Shigella $(0.956 \%$ vs. $6.577 \%, P<0.05)$ was observed in LBW piglets on D14 but a higher abundance of Moryella on D14 (0.020\% vs. $0.000 \%, P<0.05)$ and D21 $(0.059 \%$ vs. $0.014 \%, P<0.05)$ was seen in NBW piglets. On D21, an increased relative abundance of the genus Bacteroides (9.160\% vs. $3.923 \%, P<0.05$ ) was observed for LBW piglets. Meanwhile, the relative abundance of Campylobacter $(0.326 \%$ vs. $0.048 \%, P<0.05)$ increased again as that on D7. Additionally, LBW piglets had a lower proportion of the genus Howardella, both on D21 (0.008\% vs. $0.051 \%$, $P<0.05)$ and D35 (0.012\% vs. $0.055 \%, P<0.05)$, when compared with NBW piglets. As the predominant genus, the proportion of the genus Lactobacillus was continuously lower in LBW piglets than in NBW piglets from D7 to D35, with a significant decrease on D21 (0.790\% vs. $6.316 \%, P<0.05$, Supplementary Figure S4D). Compared to NBW piglets, the relative abundance of the genus Prevotella was significantly higher on D14 (26.343\% vs. $7.276 \%, P<0.05)$ and D35 (26.948\% vs. $16.736 \%, P<0.05)$ but significantly lower on D21 (8.342\% vs. $19.499 \%, P<0.05$ ) in LBW piglets (Supplementary Figure S4F). The proportion of the genus Streptococcus was low in the LBW group during the suckling period (Supplementary Figure S4H), with a significant decline on D21 $(0.087 \%$ vs. $0.243 \%, P<0.05)$.

\section{Differences in Fecal Metabolite Profiles Between LBW and NBW Piglets During Early-Life}

The fecal metabolic profiles were analyzed by UPLC-MS. Differences in the metabolite profiles of LBW and NBW piglets at each time-point were revealed by OPLS-DA (Figure 5). A total of 46 differentially abundant metabolites were identified and annotated across the whole experimental period. These metabolites, which include amino acids, organic acids, fatty acids, and lipids, are involved in multiple biological pathways (Supplementary Table S3). Compared with the NBW group, LBW piglets had higher concentrations of glycocholic acid, L-valine, and vanilpyruvic acid on D3. On D7, nine compounds (linoleic acid, $\alpha$-linolenic acid, palmitic acid, indoleacetic acid, $\alpha$-dimorphecolic acid, cyclohexane undecanoic acid, 5,8-tetradecadienoic acid, 3-oxododecanoic acid, and cyclohexanecarboxylic acid) were significantly enriched, whereas the amounts of three metabolites (N1-acetylspermine, N-undecanoylglycine, and $\mathrm{N}$-acetylcadaverine) were significantly declined in LBW piglets. It was noted that the concentrations of indoleacetic acid and $\alpha$-dimorphecolic acid in the LBW group had a 7 - and 6-fold increase, respectively. On D14, the production of ten metabolites (oleic acid, linoleic acid, kynurenic acid, indoleacetic acid, 2-phenylacetamide, deoxycholic acid, tetracosahexaenoic acid, myristoleic acid, 3-oxotetradecanoic acid, and cyclohexanecarboxylic acid) was downregulated in LBW piglets when compared with their NBW littermates. On D21, increased amounts of seven metabolites (25-hydroxycholesterol, chenodeoxycholic acid, arachidonic acid, stearoylcarnitine, zymosterol intermediate 2, docosahexaenoic acid, and desaminotyrosine) were observed in the LBW group, while three metabolites (desmosterol, phenylalanylphenylalanine, and 3-oxohexadecanoic acid) were reduced. In addition, on D35, LBW piglets had higher concentrations of etiocholanedione, 3-hydroxyhippuric acid, and L-phenylalanine. In contrast, lower values of eight metabolites (palmitic acid, L-glutamic acid, succinic acid, 3 $\beta, 7 \alpha$-dihydroxy5-cholestenoate, allolithocholic acid, N-acetylneuraminic acid, hypogeic acid, and $\mathrm{N}$-acetylserine) were found in LBW piglets. Further metabolite enrichment analysis indicated that LBW had a significant impact on fatty acid metabolism and biosynthesis, bile acid biosynthesis, and amino acid metabolism in piglets (Figure 6). 

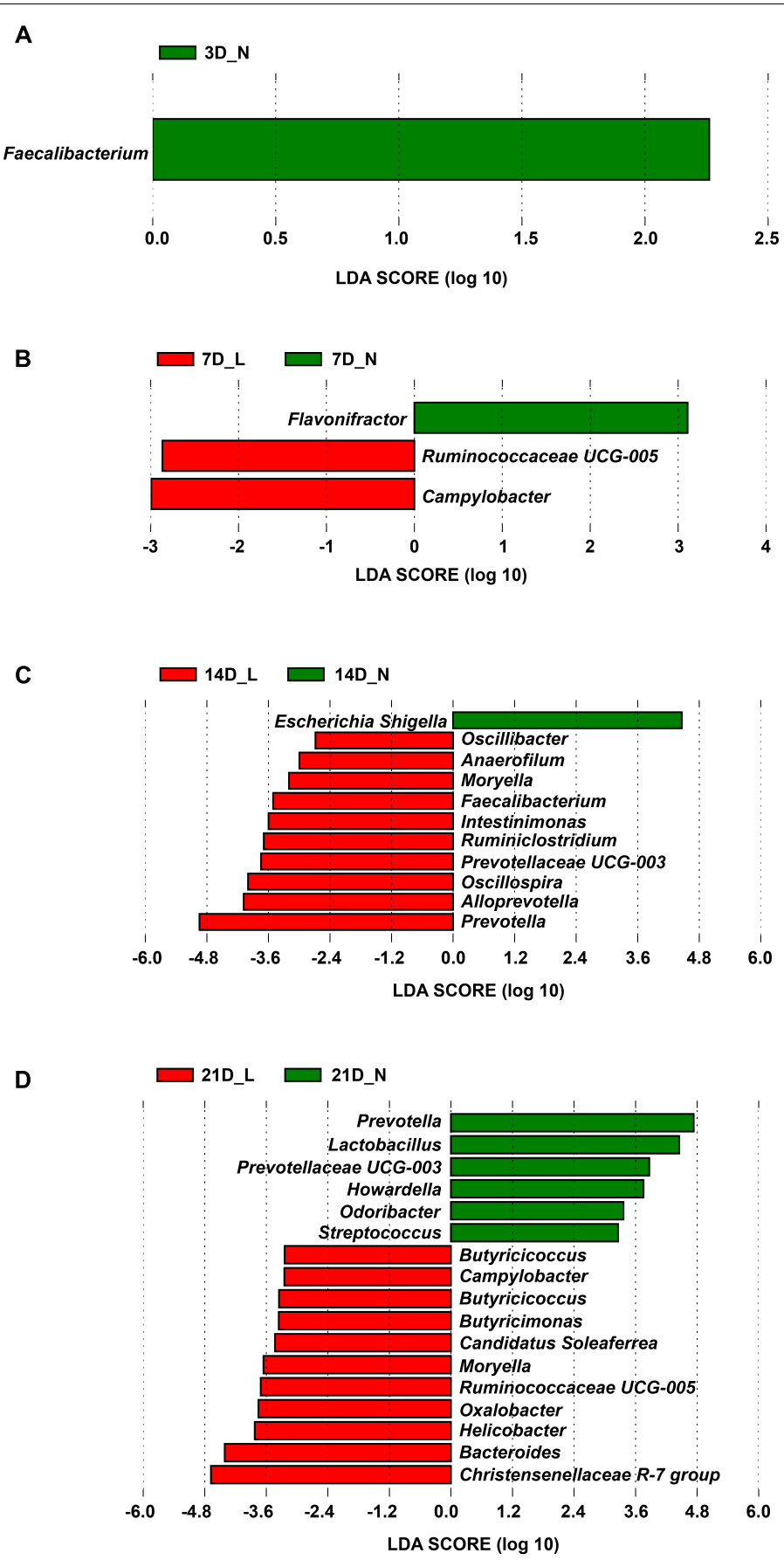

E

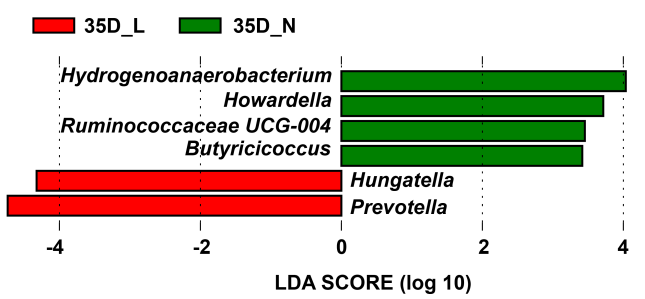

FIGURE 4 | Differentially abundant genera between LBW and NBW piglets by sampling time. Histograms of a linear discriminant analysis (LDA) score (threshold $\geq 2$ ) on D3 (A), D7 (B), D14 (C), D21 (D), and D35 (E) are plotted. $n=6$ per group. L, low-birth-weight; N, normal-birth-weight. 
Li et al.

LBW Affects Gut Microbiota Colonization

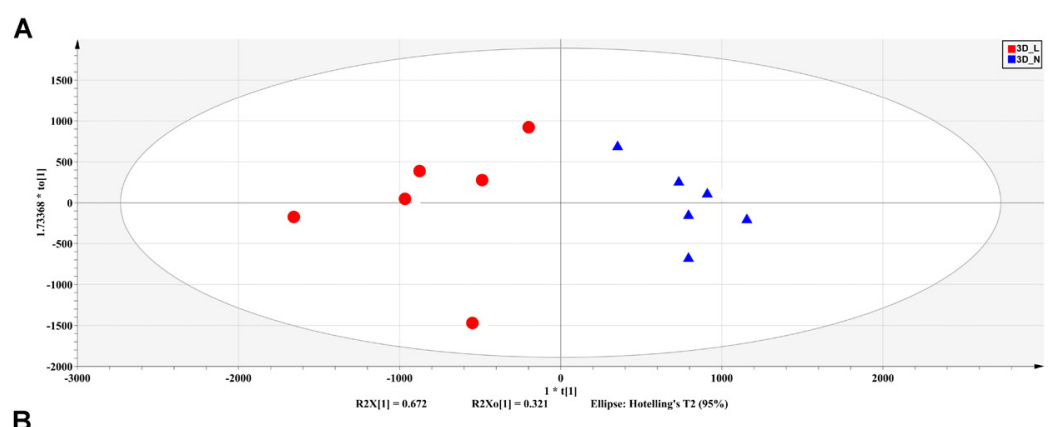

B

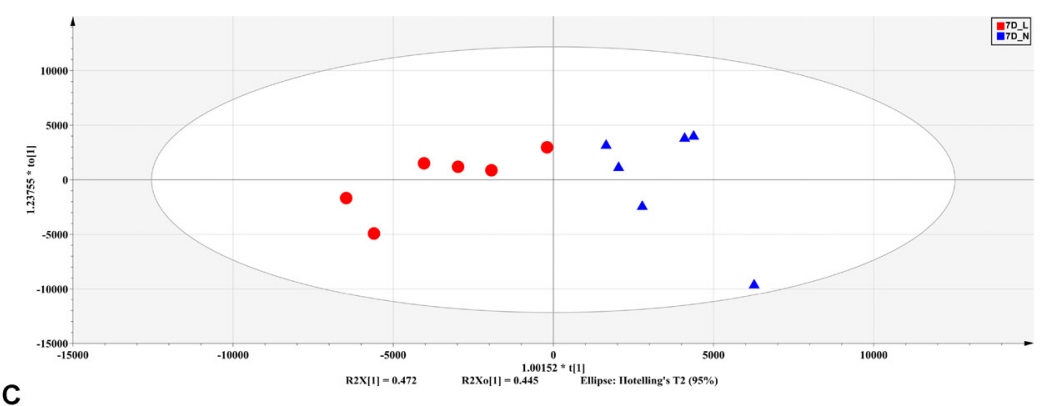

C

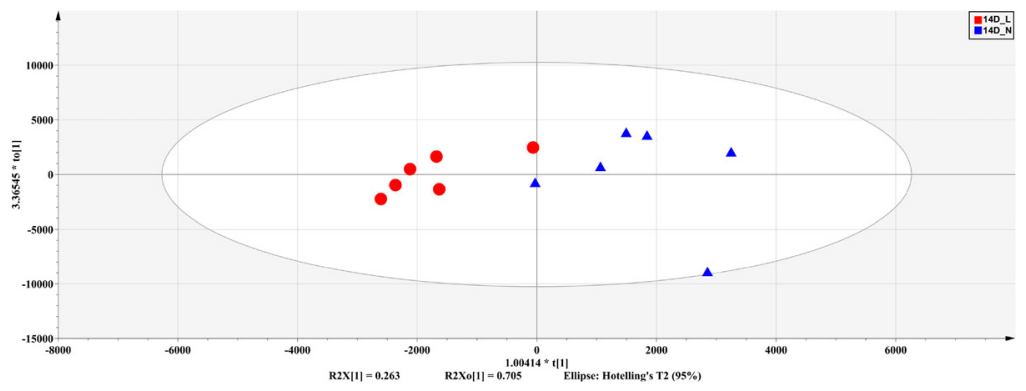

D
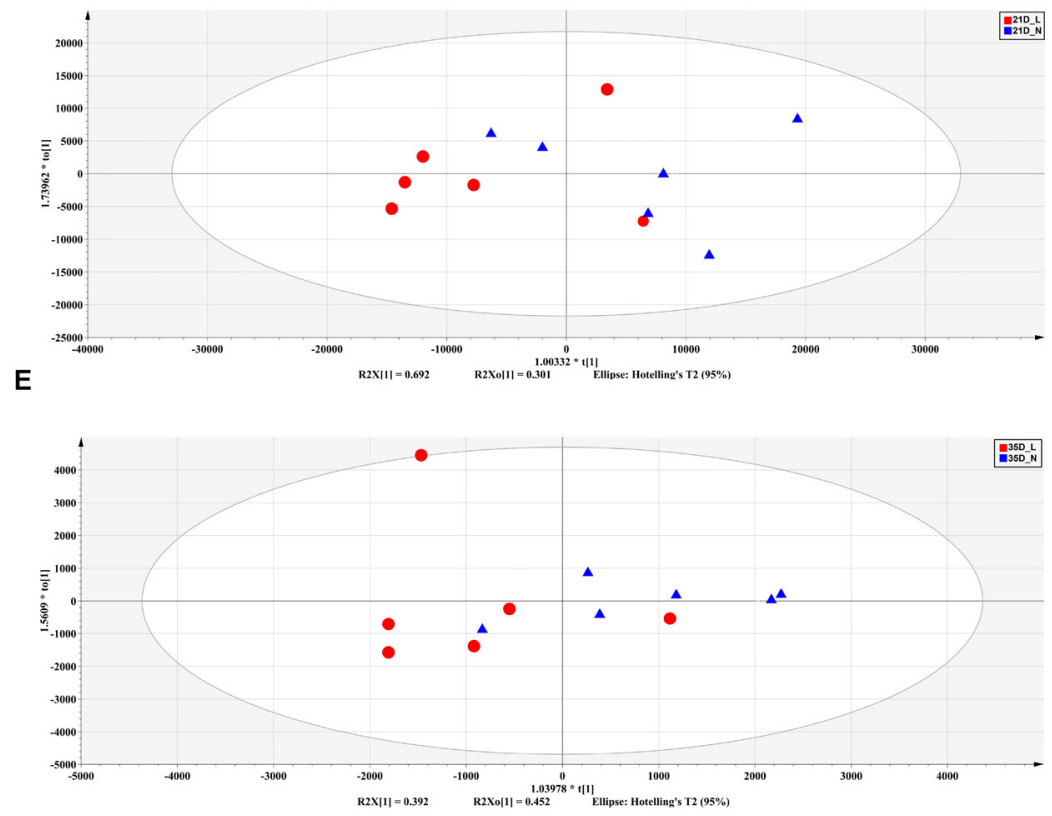

FIGURE 5 | Orthogonal partial least squares discriminant analysis (OPLS-DA) plot of fecal metabolites between LBW and NBW piglets on D3 (A), D7 (B), D14 (C), D21 (D), and D35 (E). $n=6$ per group. L, low-birth-weight; N, normal-birth-weight.

Frontiers in Microbiology | www.frontiersin.org

8

September 2018 | Volume 9 | Article 1798 


\section{Metabolite Sets Enrichment Overview}

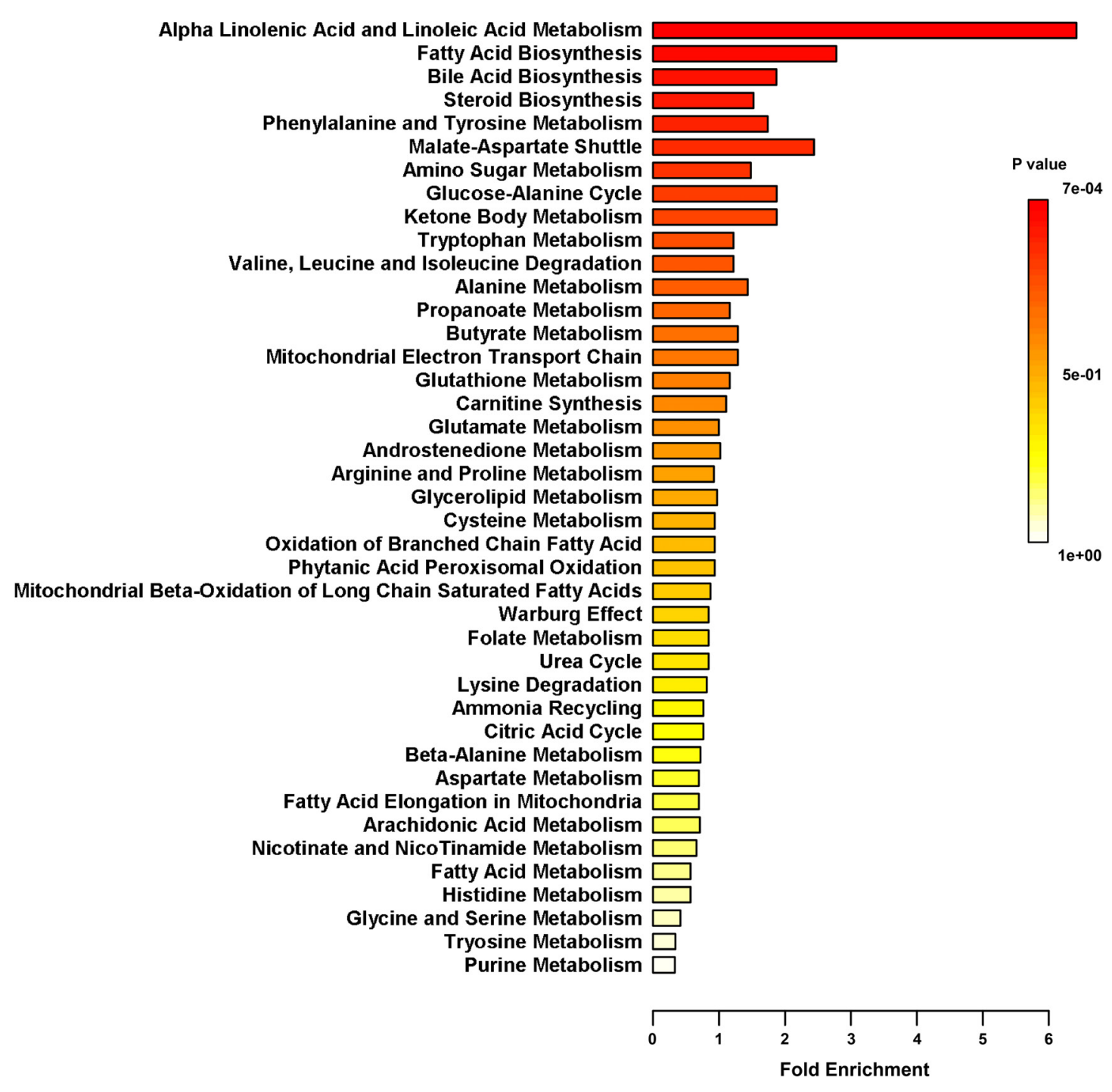

FIGURE 6 | Metabolic pathway enrichment analysis. Overview of metabolites that were enriched in LBW piglets compared to the NBW ones. $n=6$ per group.

\section{Correlations Between the Fecal Microbial Composition and Metabolite Profiles}

A Spearman's correlation matrix was generated to explore the correlation between the bacterial genera and candidate compounds that were significantly affected by birth weight. As shown in Figure 7, significant associations could be identified between the gut microbiota and the altered metabolite profiles from D7 to D35. On D7 (Figure 7A), the correlation analysis revealed that the genus Flavonifractor was positively correlated with $\mathrm{N}$-undecanoylglycine $(R=0.79, P<0.05)$. The genus Ruminococcaceae UCG-005 was negatively associated with N1Acetylspermine $(R=-0.83, P<0.05)$. The genus Campylobacter, having an increased abundance in LBW piglets, was positively correlated with indoleacetic acid $(R=0.73, P<0.05)$ and negatively associated with $\mathrm{N} 1$-acetylspermine $(R=-0.62$, $P<0.05)$ and $\mathrm{N}$-acetylcadaverine $(R=-0.90, P<0.05)$. On D14 (Figure 7B), 2-phenylacetamide was positively correlated with the genus Escherichia-Shigella $(R=0.74, P<0.05)$ and negatively associated with the genera Intestinimonas $(R=-0.63, P<0.05)$ and Faecalibacterium $(R=-0.85$, $P<0.05)$. Oleic acid and cyclohexanecarboxylic acid were negatively associated with the same nine genera $(R<-0.58$, $P<0.05$ ). These nine genera included Anaerofilum, Prevotella, Ruminiclostridium, Prevotellaceae UCG-003, Faecalibacterium, Alloprevotella, Oscillibacter, Oscillospira, Intestinimonas. Myristoleic acid was negatively associated with eight genera $(R<-0.62, P<0.05)$. Linoleic acid had negative associations with four genera $(R<-0.57, P<0.05)$, deoxycholic acid 


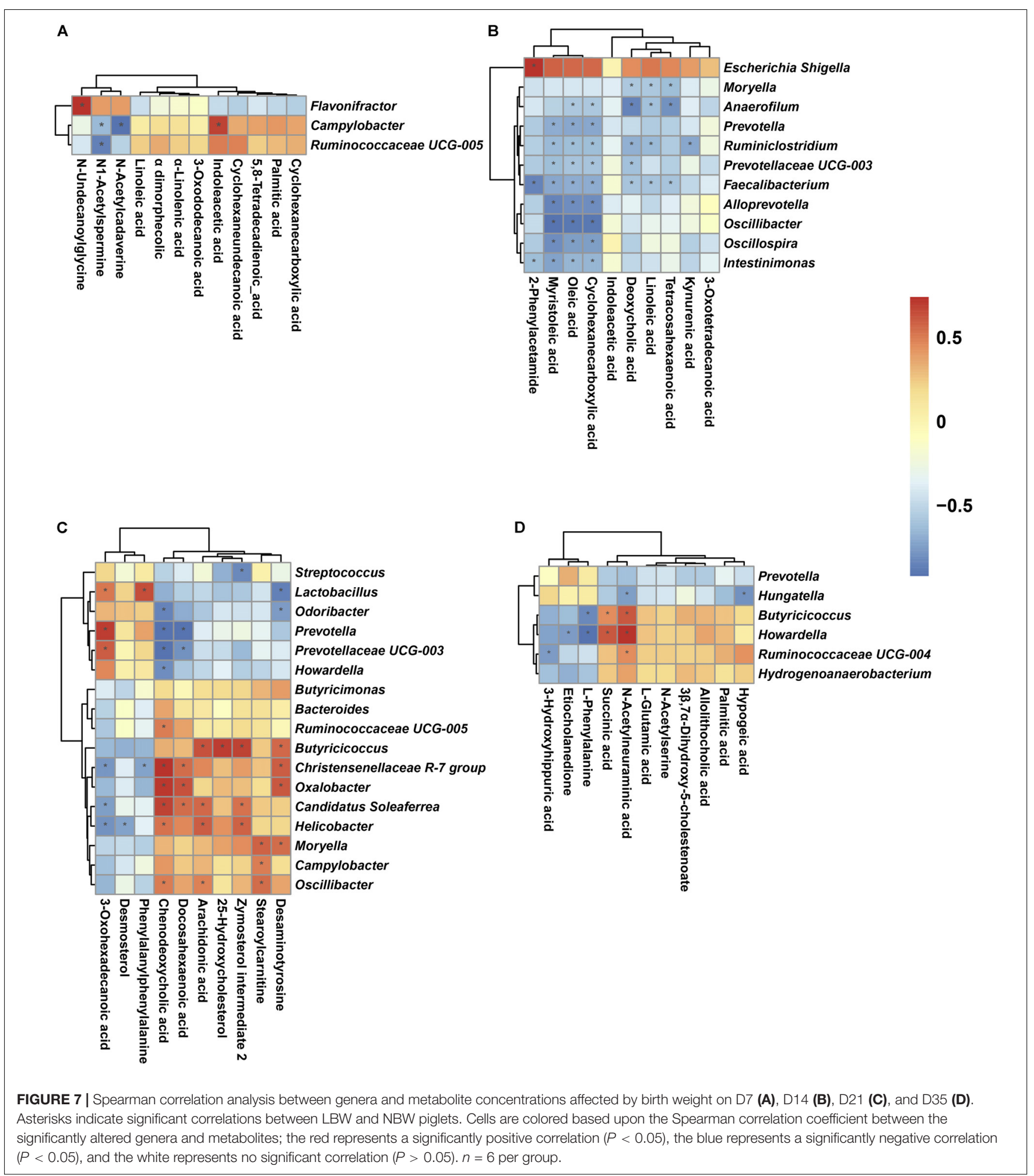

with five genera $(R<-0.57, P<0.05)$, kynurenic acid with the genus Ruminiclostridium $(R=-0.71, P<0.05)$, and tetracosahexaenoic acid with three genera $(R<-0.59, P<0.05)$. On D21 (Figure 7C), ten genera (6 positive and 4 negative) were significantly correlated with chenodeoxycholic acid $(P<0.05)$; six genera ( 3 positive and 3 negative) were associated with 3 -oxohexadecanoic acid $(P<0.05)$; five genera $(3$ positive and 2 negative) were associated with docosahexaenoic acid $(P<0.05)$; and three and four genera were positively associated with stearoylcarnitine and arachidonic acid, respectively $(R>0.58$, 
$P<0.05)$. Similarly, desaminotyrosine, zymosterol intermediate 2, desmosterol, and phenylalanylphenylalanine were significantly correlated with six genera (4 positive and 2 negative), four genera ( 3 positive and 1 negative), one genera (negative), and two genera ( 1 positive and 1 negative), respectively $(P<0.05)$. For 35-day-old piglets (Figure 7D), the genus Ruminococcaceae UCG-004 showed a negative correlation with 3-hydroxyhippuric acid $(R=-0.59, P<0.05)$ but a positive correlation with $\mathrm{N}$-acetylneuraminic acid $(R=0.60, P<0.05)$. The genus Hungatella presented negative associations with hypogeic acid and $\mathrm{N}$-acetylneuraminic acid $(R<-0.57, P<0.05)$. The genera Howardella and Butyricicoccus showed negative associations with L-phenylalanine $(R<-0.67, P<0.05)$, whereas positive connections with succinic acid and $\mathrm{N}$-acetylneuraminic acid $(R>0.59, P<0.05)$.

\section{DISCUSSION}

Low-birth-weight piglets are strongly associated with high rates of postnatal mortality, reduced growth rates, and poor carcass quality (Wu et al., 2006; Berends et al., 2013). In the current study, we confirmed that LBW piglets had continuously poor growth performance during early-life, which was consistent with previous findings (Wang et al., 2010; Douglas et al., 2014; Hu et al., 2015). In addition, we systematically identified differentially abundant bacterial genera and metabolites between LBW and NBW piglets, from birth through the postweaning period, using a microbiota-metabolome analysis. Our findings showed that the dynamic establishment of the gut microbiota was strongly affected by age, and LBW was associated with alterations in the gut microbial community and fecal metabolome of piglets during the suckling and weaning period. Furthermore, associations existed between the differentially abundant genera and metabolites in the LBW and NBW groups.

\section{Establishment of the Gut Microbial Community in Newborn Piglets}

In the present study, Firmicutes and Bacteroidetes were the two most predominant phyla in piglet gut microbiota, which was consistent with previous studies in piglets (Bian et al., 2016; Hu et al., 2016; Chen et al., 2017) and human infants (Backhed et al., 2015; Kostic et al., 2015). Fusobacteria was dominant on D3 but disappeared after weaning, which was also observed in other studies (Bian et al., 2016; Chen et al., 2017). Proteobacteria, which include a wide variety of pathogenic bacteria, also showed a significant decline with age, and this observation was in agreement with previous studies (Zhao et al., 2015; Bian et al., 2016; Hu et al., 2016; Chen et al., 2017). These results suggested that potential pathogens might be mainly present in the newborn GIT, and therefore make newborns more susceptible to disease. At the genus level, the relative abundance of Fusobacterium sharply reduced from the suckling to weaning period as reported in other studies (Chen et al., 2017). Lactobacillus was predominant in the gut microbiota of piglets from D7 to D35 in our study, which was in accordance with studies carried out by Bian et al. (2016) and Mach et al. (2015).
Bacterial richness and diversity increased over time, and this finding was in accordance with other studies in piglets (Frese et al., 2015; Mach et al., 2015; Bian et al., 2016; Chen et al., 2017) and human infants (Matamoros et al., 2013; Backhed et al., 2015; Hill et al., 2017).

\section{Differences in the Gut Microbiota Between LBW and NBW Piglets During Early-Life}

Evidence has shown that bacterial establishment in the gut can be altered in premature infants born with LBW during early-life (Fanca-Berthon et al., 2010; Arboleya et al., 2012a,b). Studies in piglets have reported greater counts of adherent bacteria in the intestinal mucosa of 2- to 5-day-old piglets with IUGR (D'Inca et al., 2010, 2011). In the current study, no clear separation was observed in the gut microbial community structure of LBW and NBW piglets on D3. Immediately after birth, neonates experience a transition from an intrauterine environment to an external environment. The fetus is in a relatively sterile aquatic environment during pregnancy and depends mainly on the umbilical vein for parenteral nutrition. Immediately after birth, the neonate begins enteral nutrition by suckling maternal milk and the GIT is colonized with microbes. Therefore, rapid changes in environment and nutrition during the early postnatal period may lead to high intraindividual variability in the gut microbiota of piglets, and these changes explain the lack of significant differences between the LBW and NBW piglets on D3. Our results revealed that the bacterial community structure of LBW and NBW piglets showed significant separation on D7, D14, and D21, but not on D35. Impaired development of the GIT (Wang et al., 2010) and insufficient milk intake (Quesnel et al., 2012) in LBW piglets, might be the two factors that explain the subsequent dissimilarity in the structure of the gut microbiota between the two groups. After weaning, the large changes in the environment and diet may result in a similar microbial community structure for both LBW and NBW piglets.

Furthermore, our findings demonstrated that birth weight affected specific bacterial taxa at all time-points, especially from D7 to D21. This suggests that the suckling period may be the critical developmental period for the gut microbiota and have long-lasting impacts on health and metabolism. A lower relative abundance of Firmicutes was harbored by LBW piglets on D3 and D7, which was in agreement with the fact that the relative abundance of Firmicutes decreased in the placenta from LBW infants (Zheng et al., 2015). Also, in the present study, LBW piglets had a lower abundance of Lactobacillus and Streptococcus but a higher abundance of Fusobacterium, which was consistent with previous studies in LBW infants (Arboleya et al., 2012a; Zheng et al., 2015) and rodents (Wang et al., 2016). LBW piglets are well recognized as being more susceptible to GIT defects and various diseases (Li et al., 2017; Wang et al., 2017). Some Fusobacterium spp. are pathogenic in pigs (De Witte et al., 2017), whereas Lactobacillus spp. are widely used as probiotics to improve health and disease resistance (Naito et al., 2011; Wang et al., 2012), and Streptococcus spp. are commensal members in the gut of newborns (Matamoros et al., 2013). Thus, the observed 
alterations in the members of these genera may partly explain the high morbidity of LBW piglets. Additionally, the relative abundances of Faecalibacterium on D3 and Prevotella on D21 were significantly decreased in the LBW group when compared with the NBW group. Prevotella spp. are important acetateproducing bacteria (Ivarsson et al., 2014) and Faecalibacterium include many key butyrate-producing species (Benus et al., 2010), and these SCFAs improve intestinal barrier function and reduce inflammation in the gut (Liu et al., 2018; Turroni et al., 2018). It has been reported that $50 \%$ of butyrate-producing bacteria are able to produce butyrate from acetate (Barcenilla et al., 2000). The decline of these SCFA-producers might reflect an attenuated capacity to metabolize dietary fiber in LBW piglets.

Campylobacter spp., some of which are associated with diarrhea in piglets, were more abundant in LBW piglets than in NBW piglets on D7 and D21 (Yang et al., 2017). Also, LBW piglets had higher levels of Moryella and Oscillibacter on D14 and D21 when compared with the NBW group in our study. A recent study reported that adult mice with obesity had a higher relative abundance of Moryella (Sung et al., 2017). Similarly, Duca et al. (2014) found that the abundance of Oscillibacter was significantly higher in obese-prone rats when compared with obese-resistant rats. Low-birth-weight neonates have a higher risk for developing adult obesity (Li et al., 2017); therefore, whether Moryella and Oscillibacter are associated with obesity in the long-term in LBW neonates needs further investigation. In our study, LBW piglets had a higher proportion of Prevotella on D14 and D35 but a lower proportion of Escherichia-Shigella on D14 when compared with NBW littermates. However, similar results were also observed in 10-day-old piglets suffering from diarrhea (Yang et al., 2017). Escherichia spp., which include many opportunistic pathogens, have been shown to be negatively correlated with Prevotella in healthy children (Kang et al., 2013), diarrheic children (Pop et al., 2014), and healthy piglets (Yang et al., 2017). However, both our observations and the studies carried out by Yang et al. (2017) showed that no significant correlation existed between these two genera in diarrheic piglets. The Escherichia-Shigella spp. detected in the present study may not be pathogenic as diarrhea was not observed in either the LBW or NBW piglets.

\section{Changes in Metabolism Between LBW and NBW Piglets During Early-Life}

In addition to alterations in the fecal microbiota of LBW piglets when compared to their NBW littermates, LBW also disrupted the metabolic status of neonatal piglets. Our metabolome data revealed that the discriminating metabolites in differentiating LBW and NBW piglets were mainly found on D7, D14, D21, and D35. At these time-points, 12, 10, 10, and 11, differential compounds in feces were identified, respectively. Similar to the observations in the bacterial community, only minor differences in metabolites were found in 3-day-old piglets, of which only three metabolites were identified.

The altered concentrations of 14 fatty acids in the feces of LBW piglets suggest a potential dysfunction in fatty acid biosynthesis and metabolism. Unsaturated and saturated fatty acids are used by the host for synthesizing triglycerides which can promote lipogenesis (Miyazaki et al., 2001). There is evidence that the levels of some metabolites associated with lipogenesis changed in neonatal piglets (He et al., 2011), fetal pigs (Lin et al., 2012), and human infants (Leduc et al., 2011) suffering from IUGR. It has been reported that LBW neonates have a higher risk for developing adult metabolic and cardiovascular disorders due to abnormal fat storage and lipid metabolism (Wu et al., 2006; Li et al., 2017). Thus, the observed alterations in these fatty acids may be an important factor in the development of metabolic diseases later in life in LBW neonates.

The concentrations of several amino acids in the feces of LBW piglets, such as L-valine, L-glutamic acid, and L-phenylalanine, were significantly different from that of NBW piglets. Amino acids serve as essential precursors to protein biosynthesis ( $\mathrm{Wu}$, 2009), and the alterations in amino acid composition indicate that body growth and development might be suppressed in LBW neonates due to the abnormality in intestinal protein synthesis (Wang et al., 2010). Specifically, we observed decreased levels of L-glutamic acid in LBW piglets on D35. Reduced concentrations of glutamine or glutamate have been reported in the small intestine of LBW piglets (He et al., 2011), umbilical plasma from LBW infants (Favretto et al., 2012; Ivorra et al., 2012), and fetal pigs (Lin et al., 2012). Glutamine plays a critical role in maintaining multiple important functions, such as nutrient metabolism, immune response, and intestinal integrity, as well as the synthesis of other bioactive compounds (Wu et al., 2007; $\mathrm{Wu}, 2010)$. Therefore, glutamine or glutamate supplementation in LBW piglets may be an effective way to improve growth and maintain gut health (Vaughn et al., 2003; Wu et al., 2011; Yuan et al., 2015). Another interesting observation in the present study is that the feces of LBW piglets have higher levels of Lphenylalanine and L-valine. However, previous studies revealed decreased levels of these two amino acids in the jejunum or umbilical plasma of LBW neonates (He et al., 2011; Favretto et al., 2012; Lin et al., 2012). These contradictory findings might have resulted from the differences in the intestinal region being sampled. Valine and phenylalanine are two indispensable amino acids for piglets and must be supplied in the diet (Lei et al., 2012; Rezaei et al., 2013); therefore, their enrichment in feces might indicate a lower bioavailability in the intestine of LBW piglets.

Additionally, we report, for first time, that birth weight status significantly alters the concentrations of several metabolites associated with bile acid biosynthesis, such as glycocholic acid, deoxycholic acid, 25-hydroxycholesterol, chenodeoxycholic acid, $3 \beta, 7 \alpha$-dihydroxy-5-cholestenoate, and allolithocholic acid. Bile acids are signaling molecules essential for the absorption and metabolism of dietary lipids and fat-soluble vitamins (Chiang, 2009). Bile acids also interact with the intestinal microbiota (Wang et al., 2002) by regulating FXR and G protein-coupled bile acid receptor 1 (GPBAR1) signaling, which in turn maintain intestinal homeostasis (Fiorucci and Distrutti, 2015). Primary bile acids are derived from cholesterol in the liver and are converted to secondary bile acids by the intestinal microbiota (Ridlon et al., 2006). In the present study, we found that LBW piglets had higher levels of primary bile acids (glycocholic acid, 25-hydroxycholesterol, and chenodeoxycholic acid) 
but lower levels of secondary bile acids (deoxycholic acid). These findings might suggest a decreased conversion rate of bile acids from the primary to the secondary form in the intestine of LBW piglets. Also, bile acids might act as an opportunistic mediator for interventions in obesity and diabetes (Fiorucci and Distrutti, 2015). Disruptions in bile acid metabolism of LBW piglets might have a long-term adverse effect on their physiological behavior and health during postnatal life.

Diet and host physiological status have direct influences on host metabolism (He et al., 2011; Maier et al., 2017). Moreover, there is increasing evidence that a part of these impacts is regulated by the intestinal microbiota as many gut microbes are capable of producing bioactive molecules that affect host metabolism (Fiorucci and Distrutti, 2015). In the current study, Spearman's correlation analysis revealed an association between the abundance of specific bacterial genera and metabolites that were significantly influenced by birth weight. The characterization of metabolic alterations modulated by the intestinal microbiota has been used to understand the molecular mechanisms of host health and disease development in humans and animals (Arrieta et al., 2015; Shi et al., 2015; Sun et al., 2016; Turroni et al., 2018). Altogether, the disruption of gut microbial composition and metabolic homeostasis could be a major underlying factor that induces stunted growth and development of LBW piglets.

\section{CONCLUSION}

In summary, the present study revealed differences in the gut microbiota and metabolic status between LBW and NBW piglets during the suckling and weaning period. Firstly, birth weight status strongly affected the gut bacterial composition of piglets from 7 to 21 days of age but had no obvious impact on D3 because of larger individual variations. This finding implies that the suckling period might be the critical period for modulating the gut microbiota in LBW piglets and interventions beginning from D7 might be more effective. Secondly, LBW piglets had altered concentrations of metabolites involved in multiple biochemical processes, including fatty acid metabolism, bile acid biosynthesis, and amino acid metabolism. Moreover, these metabolic changes in LBW piglets might be partly modulated by the intestinal microbiota as there were associations between the abundance

\section{REFERENCES}

Anderson, M. J., and Walsh, D. C. I. (2013). PERMANOVA, ANOSIM, and the mantel test in the face of heterogeneous dispersions: what null hypothesis are you testing? Ecol. Monogr. 83, 557-574. doi: 10.1890/12-2010.1

Arboleya, S., Binetti, A., Salazar, N., Fernandez, N., Solis, G., HernandezBarranco, A., et al. (2012a). Establishment and development of intestinal microbiota in preterm neonates. FEMS Microbiol. Ecol. 79, 763-772. doi: 10. 1111/j.1574-6941.2011.01261.x

Arboleya, S., Solis, G., Fernandez, N., de los Reyes-Gavilan, C. G., and Gueimonde, M. (2012b). Facultative to strict anaerobes ratio in the preterm infant microbiota: a target for intervention? Gut Microbes 3, 583-588. doi: 10.4161/gmic. 21942

Arrieta, M. C., Stiemsma, L. T., Dimitriu, P. A., Thorson, L., Russell, S., YuristDoutsch, S., et al. (2015). Early infancy microbial and metabolic alterations of specific bacterial genera and metabolites affected by birth weight. Collectively, these findings provide new directions in identifying the key factors affecting the early development of LBW piglets and formulating the corresponding nutritional intervention.

\section{AUTHOR CONTRIBUTIONS}

JW and NL designed the experiments. JW, NL, SH, and WW conducted the experiments. NL, SH, and LJ carried out the experiments and collected the samples. NL, SH, and BZ performed the analysis of samples. NL, TL, and ZL analyzed the data. NL, WW, and JW wrote and revised the manuscript. All authors read and approved the final manuscript.

\section{FUNDING}

This work was supported by the Beijing Municipal Natural Science Foundation (No. S170001), the National Natural Science Foundation of China (Nos. 31422052 and 31630074), the National Key Research and Development Program of China (2016YFD0500506 and 2018YFD0501002), the 111 Project (No. B16044), Jinxinnong Animal Science Developmental Foundation, and the Hunan Co-Innovation Center of Animal Production Safety, CICAPS.

\section{ACKNOWLEDGMENTS}

We thank Dr. Shuai Zhang for his assistance in manuscript revision and statistical analysis. We thank Dr. Ping Liu and Ms. Erica Week for their help in the manuscript preparation. We also thank the Mianyang New Hope Livestock Farming Co., Ltd., in Sichuan province, China, for their support in the pig experiments.

\section{SUPPLEMENTARY MATERIAL}

The Supplementary Material for this article can be found online at: https://www.frontiersin.org/articles/10.3389/fmicb. 2018.01798/full\#supplementary-material

affect risk of childhood asthma. Sci. Transl. Med. 7, 307ra152. doi: 10.1126/ scitranslmed.aab2271

Bacci, G., Bani, A., Bazzicalupo, M., Ceccherini, M. T., Galardini, M., Nannipieri, P., et al. (2015). Evaluation of the performances of Ribosomal Database Project (RDP) classifier for taxonomic assignment of $16 \mathrm{~s}$ rna metabarcoding sequences generated from illumina-solexa NGS. J. Genom. 3, 36-39. doi: 10.7150/jgen.9204

Backhed, F., Roswall, J., Peng, Y., Feng, Q., Jia, H., Kovatcheva-Datchary, P., et al. (2015). Dynamics and stabilization of the human gut microbiome during the first year of life. Cell Host Microbe 17, 690-703. doi: 10.1016/j.chom.2015.0 4.004

Barcenilla, A., Pryde, S. E., Martin, J. C., Duncan, S. H., Stewart, C. S., Henderson, C., et al. (2000). Phylogenetic relationships of butyrate-producing bacteria from the human gut. Appl. Environ. Microbiol. 66, 1654-1661. doi: 10.1128/AEM.66.4.1654-1661.2000 
Benjamini, Y., and Hochberg, Y. (1995). Controlling the false discovery rate: a practical and powerful approach to multiple testing. J. R. Stat. Soc. B. 57, 289-300.

Benus, R. F. J., van der Werf, T. S., Welling, G. W., Judd, P. A., Taylor, M. A., Harmsen, H. J. M., et al. (2010). Association between Faecalibacterium prausnitzii and dietary fibre in colonic fermentation in healthy human subjects. Br. J. Nutr. 104, 693-700. doi: 10.1017/S0007114510001030

Berard, J., Kreuzer, M., and Bee, G. (2008). Effect of litter size and birth weight on growth, carcass and pork quality, and their relationship to postmortem proteolysis. J. Anim. Sci. 86, 2357-2368. doi: 10.2527/jas.2008-0893

Berends, L. M., Fernandez-Twinn, D. S., Martin-Gronert, M. S., Cripps, R. L., and Ozanne, S. E. (2013). Catch-up growth following intra-uterine growthrestriction programmes an insulin-resistant phenotype in adipose tissue. Int. J. Obes. 37, 1051-1057. doi: 10.1038/ijo.2012.196

Bian, G., Ma, S., Zhu, Z., Su, Y., Zoetendal, E. G., Mackie, R., et al. (2016). Age, introduction of solid feed and weaning are more important determinants of gut bacterial succession in piglets than breed and nursing mother as revealed by a reciprocal cross-fostering model. Environ. Microbiol. 18, 1566-1577. doi: 10.1111/1462-2920.13272

Cao, J., Li, M., Chen, J., Liu, P., and Li, Z. (2016). Effects of MeJA on Arabidopsis metabolome under endogenous JA deficiency. Sci. Rep. 6:37674. doi: 10.1038/ srep37674

Caporaso, J. G., Kuczynski, J., Stombaugh, J., Bittinger, K., Bushman, F. D., Costello, E. K., et al. (2010). QIIME allows analysis of high-throughput community sequencing data. Nat. Methods 7, 335-336. doi: 10.1038/nmeth.f. 303

Chen, L., Xu, Y., Chen, X., Fang, C., Zhao, L., and Chen, F. (2017). The maturing development of gut microbiota in commercial piglets during the weaning transition. Front. Microbiol. 8:1688. doi: 10.3389/fmicb.2017.01688

Chiang, J. Y. (2009). Bile acids: regulation of synthesis. J. Lipid Res. 50, 1955-1966. doi: 10.1194/jlr.R900010-JLR200

Chong, J., Soufan, O., Li, C., Caraus, I., Li, S., Bourque, G., et al. (2018). MetaboAnalyst 4.0: towards more transparent and integrative metabolomics analysis. Nucleic Acids Res. 46, W486-W494. doi: 10.1093/nar/g ky310

De Witte, C., Flahou, B., Ducatelle, R., Smet, A., De Bruyne, E., Cnockaert, M., et al. (2017). Detection, isolation and characterization of Fusobacterium gastrosuis sp. nov. colonizing the stomach of pigs. Syst. Appl. Microbiol. 40, 42-50. doi: 10.1016/j.syapm.2016.10.001

D’Inca, R., Gras-Le Guen, C., Che, L., Sangild, P. T., and Le HuerouLuron, I. (2011). Intrauterine growth restriction delays feeding-induced gut adaptation in term newborn pigs. Neonatology 99, 208-216. doi: 10.1159/0003 14919

D’Inca, R., Kloareg, M., Gras-Le Guen, C., and Le Huerou-Luron, I. (2010). Intrauterine growth restriction modifies the developmental pattern of intestinal structure, transcriptomic profile, and bacterial colonization in neonatal pigs. J. Nutr. 140, 925-931. doi: 10.3945/jn.109.116822

Douglas, S. L., Edwards, S. A., and Kyriazakis, I. (2014). Management strategies to improve the performance of low birth weight pigs to weaning and their long-term consequences. J. Anim. Sci. 92, 2280-2288. doi: 10.2527/jas.20137388

Duca, F. A., Sakar, Y., Lepage, P., Devime, F., Langelier, B., Dore, J., et al. (2014). Replication of obesity and associated signaling pathways through transfer of microbiota from obese-prone rats. Diabetes 63, 1624-1636. doi: 10.2337/db131526

Edgar, R. C. (2013). UPARSE: highly accurate OTU sequences from microbial amplicon reads. Nat. Methods 10, 996-998. doi: 10.1038/nmeth.2604

Edgar, R. C., Haas, B. J., Clemente, J. C., Quince, C., and Knight, R. (2011). UCHIME improves sensitivity and speed of chimera detection. Bioinformatics 27, 2194-2200. doi: 10.1093/bioinformatics/btr381

Fanca-Berthon, P., Hoebler, C., Mouzet, E., David, A., and Michel, C. (2010). Intrauterine growth restriction not only modifies the cecocolonic microbiota in neonatal rats but also affects its activity in young adult rats. J. Pediatr. Gastroenterol. Nutr. 51, 402-413. doi: 10.1097/MPG.0b013e3181d7 $5 \mathrm{~d} 52$

Favretto, D., Cosmi, E., Ragazzi, E., Visentin, S., Tucci, M., Fais, P., et al. (2012). Cord blood metabolomic profiling in intrauterine growth restriction. Anal. Bioanal. Chem. 402, 1109-1121. doi: 10.1007/s00216-011-5540-z
Fiorucci, S., and Distrutti, E. (2015). Bile acid-activated receptors, intestinal microbiota, and the treatment of metabolic disorders. Trends Mol. Med. 21, 702-714. doi: 10.1016/j.molmed.2015.09.001

Foxx-Orenstein, A. E., and Chey, W. D. (2012). Manipulation of the gut microbiota as a novel treatment strategy for gastrointestinal disorders. Am. J. Gastroenterol. Suppl. 1, 41-46. doi: 10.1038/ajgsup.2012.8

Frese, S. A., Parker, K., Calvert, C. C., and Mills, D. A. (2015). Diet shapes the gut microbiome of pigs during nursing and weaning. Microbiome 3:28. doi: 10.1186/s40168-015-0091-8

Goodrich, J. K., Waters, J. L., Poole, A. C., Sutter, J. L., Koren, O., Blekhman, R., et al. (2014). Human genetics shape the gut microbiome. Cell 159, 789-799. doi: 10.1016/j.cell.2014.09.053

He, Q., Ren, P., Kong, X., Xu, W., Tang, H., Yin, Y., et al. (2011). Intrauterine growth restriction alters the metabonome of the serum and jejunum in piglets. Mol. Biosyst. 7, 2147-2155. doi: 10.1039/c1mb05024a

Hill, C. J., Lynch, D. B., Murphy, K., Ulaszewska, M., Jeffery, I. B., O’Shea, C. A., et al. (2017). Erratum to: evolution of gut microbiota composition from birth to 24 weeks in the INFANTMET Cohort. Microbiome 5:21. doi: 10.1186/s40168017-0240-3

Hong, X., Chen, J., Liu, L., Wu, H., Tan, H., Xie, G., et al. (2016). Metagenomic sequencing reveals the relationship between microbiota composition and quality of Chinese Rice Wine. Sci. Rep. 6:26621. doi: 10.1038/srep26621

Hu, J., Nie, Y., Chen, J., Zhang, Y., Wang, Z., Fan, Q., et al. (2016). Gradual changes of gut microbiota in weaned miniature piglets. Front. Microbiol. 7:1727. doi: $10.3389 /$ fmicb.2016.01727

Hu, L., Liu, Y., Yan, C., Peng, X., Xu, Q., Xuan, Y., et al. (2015). Postnatal nutritional restriction affects growth and immune function of piglets with intra-uterine growth restriction. Br. J. Nutr. 114, 53-62. doi: 10.1017/S0007114515001579

Inman, C. F., Haverson, K., Konstantinov, S. R., Jones, P. H., Harris, C., Smidt, H., et al. (2010). Rearing environment affects development of the immune system in neonates. Clin. Exp. Immunol. 160, 431-439. doi: 10.1111/j.1365-2249.2010. 04090. $x$

Ivarsson, E., Roos, S., Liu, H. Y., and Lindberg, J. E. (2014). Fermentable non-starch polysaccharides increases the abundance of Bacteroides-PrevotellaPorphyromonas in ileal microbial community of growing pigs. Animal 8, 1777-1787. doi: 10.1017/S1751731114001827

Ivorra, C., Garcia-Vicent, C., Chaves, F. J., Monleon, D., Morales, J. M., and Lurbe, E. (2012). Metabolomic profiling in blood from umbilical cords of low birth weight newborns. J. Transl. Med. 10:142. doi: 10.1186/1479-5876-10-142

Kabat, A. M., Srinivasan, N., and Maloy, K. J. (2014). Modulation of immune development and function by intestinal microbiota. Trends Immunol. 35, 507517. doi: 10.1016/j.it.2014.07.010

Kang, D. W., Park, J. G., Ilhan, Z. E., Wallstrom, G., Labaer, J., Adams, J. B., et al. (2013). Reduced incidence of Prevotella and other fermenters in intestinal microflora of autistic children. PLoS One 8:e68322. doi: 10.1371/journal.pone. 0068322

Kostic, A. D., Gevers, D., Siljander, H., Vatanen, T., Hyotylainen, T., Hamalainen, A. M., et al. (2015). The dynamics of the human infant gut microbiome in development and in progression toward type 1 diabetes. Cell Host Microbe 17, 260-273. doi: 10.1016/j.chom.2015.01.001

Leduc, L., Delvin, E., Ouellet, A., Garofalo, C., Grenier, E., Morin, L., et al. (2011). Oxidized low-density lipoproteins in cord blood from neonates with intrauterine growth restriction. Eur. J. Obstet. Gynecol. Reprod. Biol. 156, 46-49. doi: 10.1016/j.ejogrb.2011.01.007

Lei, J., Feng, D., Zhang, Y., Zhao, F. Q., Wu, Z., San Gabriel, A., et al. (2012). Nutritional and regulatory role of branched-chain amino acids in lactation. Front. Biosci. 17, 2725-2739. doi: 10.2741/4082

Li, M., Bauer, L. L., Chen, X., Wang, M., Kuhlenschmidt, T. B., Kuhlenschmidt, M. S., et al. (2012). Microbial composition and in vitro fermentation patterns of human milk oligosaccharides and prebiotics differ between formula-fed and sow-reared piglets. J. Nutr. 142, 681-689. doi: 10.3945/jn.111.154427

Li, N., Wang, W., Wu, G., and Wang, J. (2017). Nutritional support for low birth weight infants: insights from animal studies. Br. J. Nutr. 117, 1390-1402. doi: 10.1017/S000711451700126X

Lin, G., Liu, C., Feng, C., Fan, Z., Dai, Z., Lai, C., et al. (2012). Metabolomic analysis reveals differences in umbilical vein plasma metabolites between normal and growth-restricted fetal pigs during late gestation. J. Nutr. 142, 990-998. doi: $10.3945 /$ jn.111.153411 
Liu, H., Wang, J., He, T., Becker, S., Zhang, G., Li, D., et al. (2018). Butyrate: a double-edged sword for health? Adv. Nutr. 9, 21-29. doi: 10.1093/advances/ nmx009

Mach, N., Berri, M., Estelle, J., Levenez, F., Lemonnier, G., Denis, C., et al. (2015). Early-life establishment of the swine gut microbiome and impact on host phenotypes. Environ. Microbiol. Rep. 7, 554-569. doi: 10.1111/1758-2229. 12285

Maier, T. V., Lucio, M., Lee, L. H., VerBerkmoes, N. C., Brislawn, C. J., Bernhardt, J., et al. (2017). Impact of dietary resistant starch on the human gut microbiome, metaproteome, and metabolome. mBio 8:e01343-17. doi: 10.1128/ mBio.01343-17

Masella, A. P., Bartram, A. K., Truszkowski, J. M., Brown, D. G., and Neufeld, J. D. (2012). PANDAseq: paired-end assembler for illumina sequences. BMC Bioinformatics 13:31. doi: 10.1186/1471-2105-13-31

Matamoros, S., Gras-Leguen, C., Le Vacon, F., Potel, G., and de La Cochetiere, M. F. (2013). Development of intestinal microbiota in infants and its impact on health. Trends Microbiol. 21, 167-173. doi: 10.1016/j.tim.2012.12.001

Miyazaki, M., Kim, Y. C., and Ntambi, J. M. (2001). A lipogenic diet in mice with a disruption of the stearoyl-CoA desaturase 1 gene reveals a stringent requirement of endogenous monounsaturated fatty acids for triglyceride synthesis. J. Lipid Res. 42, 1018-1024.

Mu, C., Yang, Y., Su, Y., Zoetendal, E. G., and Zhu, W. (2017). Differences in microbiota membership along the gastrointestinal tract of piglets and their differential alterations following an early-life antibiotic intervention. Front. Microbiol. 8:797. doi: 10.3389/fmicb.2017.00797

Naito, E., Yoshida, Y., Makino, K., Kounoshi, Y., Kunihiro, S., Takahashi, R., et al. (2011). Beneficial effect of oral administration of Lactobacillus casei strain Shirota on insulin resistance in diet-induced obesity mice. J. Appl. Microbiol. 110, 650-657. doi: 10.1111/j.1365-2672.2010.04922.x

Pop, M., Walker, A. W., Paulson, J., Lindsay, B., Antonio, M., Hossain, M. A., et al. (2014). Diarrhea in young children from low-income countries leads to largescale alterations in intestinal microbiota composition. Genome Biol. 15:R76. doi: 10.1186/gb-2014-15-6-r76

Pruesse, E., Quast, C., Knittel, K., Fuchs, B. M., Ludwig, W., Peplies, J., et al. (2007). SILVA: a comprehensive online resource for quality checked and aligned ribosomal RNA sequence data compatible with ARB. Nucleic Acids Res. 35 , 7188-7196. doi: 10.1093/nar/gkm864

Quesnel, H., Brossard, L., Valancogne, A., and Quiniou, N. (2008). Influence of some sow characteristics on within-litter variation of piglet birth weight. Animal 2, 1842-1849. doi: 10.1017/S175173110800308X

Quesnel, H., Farmer, C., and Devillers, N. (2012). Colostrum intake: influence on piglet performance and factors of variation. Livest. Sci. 146, 105-114. doi: 10.1016/j.livsci.2012.03.010

Rezaei, R., Wang, W., Wu, Z., Dai, Z., Wang, J., and Wu, G. (2013). Biochemical and physiological bases for utilization of dietary amino acids by young Pigs. J. Anim. Sci. Biotechnol. 4:7. doi: 10.1186/2049-1891-4-7

Ridlon, J. M., Kang, D. J., and Hylemon, P. B. (2006). Bile salt biotransformations by human intestinal bacteria. J. Lipid Res. 47, 241-259. doi: 10.1194/jlr. R500013-JLR200

Rooks, M. G., and Garrett, W. S. (2016). Gut microbiota, metabolites and host immunity. Nat. Rev. Immunol. 16, 341-352. doi: 10.1038/nri.2 016.42

Saavedra, J. M., and Dattilo, A. M. (2012). Early development of intestinal microbiota: implications for future health. Gastroenterol. Clin. North. Am. 41, 717-731. doi: 10.1016/j.gtc.2012.08.001

Schloss, P. D., Westcott, S. L., Ryabin, T., Hall, J. R., Hartmann, M., Hollister, E. B., et al. (2009). Introducing mothur: open-source, platform-independent, community-supported software for describing and comparing microbial communities. Appl. Environ. Microbiol. 75, 7537-7541. doi: 10.1128/AEM. 01541-09

Schokker, D., Zhang, J., Zhang, L. L., Vastenhouw, S. A., Heilig, H. G., Smidt, H., et al. (2014). Early-life environmental variation affects intestinal microbiota and immune development in new-born piglets. PLoS One 9:e100040. doi: 10.1371/ journal.pone.0100040

Shi, X., Wei, X., Yin, X., Wang, Y., Zhang, M., Zhao, C., et al. (2015). Hepatic and fecal metabolomic analysis of the effects of Lactobacillus rhamnosus GG on alcoholic fatty liver disease in mice. J. Proteome Res. 14, 1174-1182. doi: $10.1021 / \mathrm{pr} 501121 \mathrm{c}$
Sun, Y., Su, Y., and Zhu, W. (2016). Microbiome-metabolome responses in the cecum and colon of pig to a high resistant starch diet. Front. Microbiol. 7:779. doi: $10.3389 /$ fmicb.2016.00779

Sung, M. M., Kim, T. T., Denou, E., Soltys, C. L. M., Hamza, S. M., Byrne, N. J., et al. (2017). Improved glucose homeostasis in obese mice treated with resveratrol is associated with alterations in the gut microbiome. Diabetes Metab. Res. Rev. 66, 418-425. doi: 10.2337/db16-0680

Turroni, S., Brigidi, P., Cavalli, A., and Candela, M. (2018). Microbiota-host transgenomic metabolism, bioactive molecules from the inside. J. Med. Chem. 61, 47-61. doi: 10.1021/acs.jmedchem.7b00244

Vaughn, P., Thomas, P., Clark, R., and Neu, J. (2003). Enteral glutamine supplementation and morbidity in low birth weight infants. J. Pediatr. 142, 662-668. doi: 10.1067/mpd.2003.208

Wang, J., Chen, L., Li, D., Yin, Y., Wang, X., Li, P., et al. (2008). Intrauterine growth restriction affects the proteomes of the small intestine, liver, and skeletal muscle in newborn pigs. J. Nutr. 138, 60-66. doi: 10.1093/jn/138.1.60

Wang, J., Feng, C., Liu, T., Shi, M., Wu, G., and Bazer, F. W. (2017). Physiological alterations associated with intrauterine growth restriction in fetal pigs: causes and insights for nutritional optimization. Mol. Reprod. Dev. 84, 897-904. doi: $10.1002 / \mathrm{mrd} .22842$

Wang, J., Tang, H., Wang, X., Zhang, X., Zhang, C., Zhang, M., et al. (2016). The structural alteration of gut microbiota in low-birth-weight mice undergoing accelerated postnatal growth. Sci. Rep. 6:27780. doi: 10.1038/srep27780

Wang, L., Lee, Y. K., Bundman, D., Han, Y., Thevananther, S., Kim, C. S., et al. (2002). Redundant pathways for negative feedback regulation of bile acid production. Dev. Cell. 2, 721-731. doi: 10.1016/S1534-5807(02)00187-9

Wang, M., Radlowski, E. C., Monaco, M. H., Fahey, G. C. Jr., Gaskins, H. R., and Donovan, S. M. (2013). Mode of delivery and early nutrition modulate microbial colonization and fermentation products in neonatal piglets. J. Nutr. 143, 795-803. doi: 10.3945/jn.112.173096

Wang, Q., Dong, J., and Zhu, Y. (2012). Probiotic supplement reduces risk of necrotizing enterocolitis and mortality in preterm very low-birth-weight infants: an updated meta-analysis of 20 randomized, controlled trials. J. Pediatr. Surg. 47, 241-248. doi: 10.1016/j.jpedsurg.2011.09.064

Wang, X., Lin, G., Liu, C., Feng, C., Zhou, H., Wang, T., et al. (2014). Temporal proteomic analysis reveals defects in small-intestinal development of porcine fetuses with intrauterine growth restriction. J. Nutr. Biochem. 25, 785-795. doi: 10.1016/j.jnutbio.2014.03.008

Wang, X., Wu, W., Lin, G., Li, D., Wu, G., and Wang, J. (2010). Temporal proteomic analysis reveals continuous impairment of intestinal development in neonatal piglets with intrauterine growth restriction. J. Proteome Res. 9, 924-935. doi: 10.1021/pr900747d

Wu, G. (2010). Functional amino acids in growth, reproduction, and health. Adv. Nutr. 1, 31-37. doi: 10.3945/an.110.1008

Wu, G., Bazer, F. W., Davis, T. A., Jaeger, L. A., Johnson, G. A., Kim, S. W., et al. (2007). Important roles for the arginine family of amino acids in swine nutrition and production. Livest. Sci. 112, 8-22. doi: 10.1016/j.livsci.2007.07.003

Wu, G., Bazer, F. W., Johnson, G. A., Knabe, D. A., Burghardt, R. C., Spencer, T. E., et al. (2011). Triennial growth symposium: important roles for l-glutamine in swine nutrition and production. J. Anim. Sci. 89, 2017-2030. doi: 10.2527/jas. 2010-3614

Wu, G., Bazer, F. W., Wallace, J. M., and Spencer, T. E. (2006). Board-invited review: intrauterine growth retardation: implications for the animal sciences. J. Anim. Sci. 84, 2316-2337. doi: 10.2527/jas.2006-156

Wu, G. Y. (2009). Amino acids: metabolism, functions, and nutrition. Amino Acids 37, 1-17. doi: 10.1007/s00726-009-0269-0

Yang, Q., Huang, X., Zhao, S., Sun, W., Yan, Z., Wang, P., et al. (2017). Structure and function of the fecal microbiota in diarrheic neonatal piglets. Front. Microbiol. 8:502. doi: 10.3389/fmicb.2017.00502

Yuan, T. L., Zhu, Y. H., Shi, M., Li, T. T., Li, N., Wu, G. Y., et al. (2015). Within-litter variation in birth weight: impact of nutritional status in the sow. J. Zhejiang Univ. Sci. B 16, 417-435. doi: 10.1631/jzus.B1500010

Zhao, W., Wang, Y., Liu, S., Huang, J., Zhai, Z., He, C., et al. (2015). The dynamic distribution of porcine microbiota across different ages and gastrointestinal tract segments. PLoS One 10:e0117441. doi: 10.1371/journal.pone.0117441

Zheng, J., Xiao, X., Zhang, Q., Mao, L., Yu, M., and Xu, J. (2015). The placental microbiome varies in association with low birth weight in full-term neonates. Nutrients 7, 6924-6937. doi: 10.3390/nu7085315 
Zmora, N., Bashiardes, S., Levy, M., and Elinav, E. (2017). The role of the immune system in metabolic health and disease. Cell Metab. 25, 506-521. doi: 10.1016/j. cmet.2017.02.006

Conflict of Interest Statement: The authors declare that the research was conducted in the absence of any commercial or financial relationships that could be construed as a potential conflict of interest.
Copyright (c) 2018 Li, Huang, Jiang, Wang, Li, Zuo, Li and Wang. This is an openaccess article distributed under the terms of the Creative Commons Attribution License (CC BY). The use, distribution or reproduction in other forums is permitted, provided the original author(s) and the copyright owner(s) are credited and that the original publication in this journal is cited, in accordance with accepted academic practice. No use, distribution or reproduction is permitted which does not comply with these terms. 